\title{
Musical Training Induces Functional Plasticity in Human Hippocampus
}

\author{
Marcus Herdener, ${ }^{1}$ Fabrizio Esposito, ${ }^{2,3}$ Francesco di Salle, ${ }^{3}$ Christian Boller, ${ }^{4}$ Caroline C. Hilti, ${ }^{1}$ Benedikt Habermeyer, ${ }^{6}$ \\ Klaus Scheffler, ${ }^{4}$ Stephan Wetzel, ${ }^{5}$ Erich Seifritz, ${ }^{1,7,8}$ and Katja Cattapan-Ludewig1,7 \\ 'University Hospital of Psychiatry, University of Bern, 3000 Bern, Switzerland, 2Department of Neuroscience, University of Naples "Federico II," 80131 \\ Naples, Italy, ${ }^{3}$ Department of Cognitive Neuroscience, University of Maastricht, 6200 MD Maastricht, The Netherlands, ${ }^{4}$ Division of Radiological Physics, \\ Institute of Radiology, University of Basel Hospital, and ${ }^{5}$ Department of Medical Radiology, Institute of Neuroradiology, University of Basel Hospital, 4031 \\ Basel, Switzerland, ${ }^{6}$ Department of Psychiatry, University of Basel, 4025 Basel, Switzerland, 7Sanatorium Kilchberg, 8802 Kilchberg, Switzerland, and \\ ${ }^{8}$ Psychiatric University Hospital, University of Zurich, 8032 Zurich, Switzerland
}

Training can change the functional and structural organization of the brain, and animal models demonstrate that the hippocampus formation is particularly susceptible to training-related neuroplasticity. In humans, however, direct evidence for functional plasticity of the adult hippocampus induced by training is still missing. Here, we used musicians' brains as a model to test for plastic capabilities of the adult human hippocampus. By using functional magnetic resonance imaging optimized for the investigation of auditory processing, we examined brain responses induced by temporal novelty in otherwise isochronous sound patterns in musicians and musical laypersons, since the hippocampus has been suggested previously to be crucially involved in various forms of novelty detection. In the first crosssectional experiment, we identified enhanced neural responses to temporal novelty in the anterior left hippocampus of professional musicians, pointing to expertise-related differences in hippocampal processing. In the second experiment, we evaluated neural responses to acoustic temporal novelty in a longitudinal approach to disentangle training-related changes from predispositional factors. For this purpose, we examined an independent sample of music academy students before and after two semesters of intensive aural skills training. After this training period, hippocampal responses to temporal novelty in sounds were enhanced in musical students, and statistical interaction analysis of brain activity changes over time suggests training rather than predisposition effects. Thus, our results provide direct evidence for functional changes of the adult hippocampus in humans related to musical training.

\section{Introduction}

The ability to make music to a professional standard implies a high degree of performance, which is acquired after years of intensive training and is one of the most complex human achievements involving various brain regions (Peretz, 2006). The musician's brain is thus regarded as a suitable model to study neuroplastic changes (Munte et al., 2002). However, the effect of musical expertise acquired through years of intensive training on functional properties of the hippocampus remained elusive, although animal models show that the hippocampus formation is particularly susceptible to neuroplastic changes modulated by various environmental factors and learning processes (Kempermann et al., 1997; Lledo et al., 2006).

Hippocampal plasticity in humans in general has mainly been inferred indirectly, by measuring the structural changes of this

\footnotetext{
Received Sept. 11, 2009; revised Nov. 17, 2009; accepted Dec. 1, 2009.

This work was supported by the Stiftung für klinische neuropsychiatrische Forschung, Bern, Switzerland. We thank Elke Hofmann (Hochschule für Musik, Basel, Switzerland) and Hans Peter Weber (Schola Cantorum, Basel, Switzerland) for their help in recruiting professional musicians and students from the "Musik-Akademie Basel" for evaluating the aural skills of music students with music dictation and, together with Felipe Cattapan, for helpful discussions.

Correspondence should be addressed to Dr. Marcus Herdener, Max Planck Institute for Biological Cybernetics, Spemannstrasse 41, 72076 Tübingen, Germany. E-mail: marcus.herdener@tuebingen.mpg.de.

D0I:10.1523/JNEUROSCI.4513-09.2010

Copyright $\odot 2010$ the authors $\quad 0270-6474 / 10 / 301377-08 \$ 15.00 / 0$
}

region with volumetric magnetic resonance imaging and relating it to training tasks involving memory functions (Maguire et al., 2000; Draganski et al., 2006). However, evidence for altered functional measures directly reflecting changes in hippocampal processing as induced by environmental factors or training is missing.

In addition to its outstanding role for memory and spatial navigation (Maguire, 2001; Ekstrom et al., 2003), the hippocampus has been suggested to be involved in novelty detection (Knight, 1996; Strange et al., 1999). Hippocampal novelty detection is based on a comparison of actual sensory inputs with stored stimulus patterns (Gray and Rawlins, 1986; Strange and Dolan, 2001; Vinogradova, 2001; Kumaran and Maguire, 2007a).

Music consists of precisely patterned sequences of sounds, and the ability to identify isochronous temporal intervals (and temporal variations) and to synchronize precisely with sensory information is a prerequisite for playing in an ensemble. A finetuning of aural skills in professional musicians is achieved by a sophisticated ear training that musical students receive during their academic education and is regarded as crucial component of their vocational formation.

We thus hypothesized that the hippocampus might be critically involved in detection of novelty of temporal structure in the auditory domain and that training of aural skills could modulate 
the detection of temporal novelty of acoustic signals in the hippocampus.

We used functional magnetic resonance imaging (fMRI) to test these two hypotheses in two independent experiments. In a first experiment (experiment 1), by presenting an acoustic temporal mismatch paradigm (see Fig. 1) to subjects with different backgrounds of musical training (professional musicians and musical laypersons), we aimed at testing for the involvement of hippocampus in acoustic novelty detection and its putative functional modulation by musical experience using a cross-sectional design. A cross-sectional design, however, leaves open the fundamental question whether observed differences between groups are related to talent or training. Thus, in a second experiment (experiment 2) using a longitudinal design, we examined an independent sample of music academy students before and after academic aural skills training in comparison with students of nonmusical faculties to specify the impact of musical training on hippocampal acoustic novelty detection.

More generally, by investigating auditory novelty detection in musicians' brains, we aimed at studying whether the adult human hippocampus is subject to functional plasticity induced by training.

\section{Materials and Methods}

Both experiments have been approved by the local ethics committee, and all subjects provided written informed consent to participate.

\section{Subjects}

In the cross-sectional experiment, we examined seven professional musicians who were professional experts in ear training and seven nonmusicians (for subject characterization, see supplemental Table 1, available at www.jneurosci.org as supplemental material), matched for age and gender. Most of the musicians worked as lecturers at a music academy. In the longitudinal experiment, 19 musical students and 21 control students of other (nonmusical) faculties were examined (supplemental Table 2, available at www.jneurosci.org as supplemental material). The musical students were recruited from the "Musik-Akademie Basel (Hochschule fuer Musik and Schola Cantorum Basiliensis)"; the students of other faculties were recruited at the University of Basel (Basel, Switzerland).

The musical students of this study were at the beginning of their academic music studies at Hochschule fuer Musik, Basel, or at the Schola Cantorum Basiliensis. The students underwent intensive ear training with at least three semester hours lessons per week in ear training (see also supplemental material, available at www.jneurosci.org). The students were also requested by their lecturers to improve their aural skills by training at home. In our study, we tested the musical students two times, at the beginning and at the end of their first two semesters of the ear training. The mean time interval between the two test sessions was $217 \mathrm{~d}$ (SD, 16).

Students from other faculties, who, however, did not receive any aural skill training between the two longitudinal evaluation time points in addition to the formal education specific to their individual fields of study, served as a matched control group (mean interval between sessions, $198 \mathrm{~d}$; SD, 20).

\section{Auditory stimulation during functional imaging}

Subjects (experiments 1 and 2) were exposed to an acoustic temporal mismatch paradigm [similar to that of Rüsseler et al. (2001)] via MRcompatible headphones (Commander XG; Resonance Technology). In detail, sine tones (50 ms duration; $5 \mathrm{~ms}$ linear rise and fall times; carrier frequency, $1 \mathrm{kHz}$ ) were adjusted in amplitude according to the subjects' subjective feedback of sounds being clearly distinguishable from scanner noise background while not being experienced as unpleasant [no significant group differences in sound pressure level (SPL); experiment 1: mean SPL, $89 \mathrm{~dB} ; t$ test $(\mathrm{df} 12), p=0.17$; experiment 2 : mean SPL, 83.5 $\mathrm{dB}$; ANOVA, $p=0.43$ ]. The stimuli were presented with a standard stimulus onset asynchrony (SOA) of $150 \mathrm{~ms}$. Shorter SOAs with three degrees of deviance (142, 130, and $100 \mathrm{~ms}$ ) (see Fig. 1) (example audio files are presented in the supplemental material, available at www. jneurosci.org) occurred every 16-20 s, representing the novel deviant temporal structure of acoustic input. In other words, on average, 1 of 120 sine tones represented temporal novelty. Each deviant condition was repeated 12 times in a pseudorandomized order (experimental duration in total, $11 \mathrm{~min} 26 \mathrm{~s}$ ). To avoid activation caused by response selection, planning, or working memory, subjects were not required to make any sort of response during the experiment. Moreover, they were instructed to watch a silent movie and to ignore the sounds presented binaurally via headphones. This way, subjects performed an incidental task so as to avoid any explicit judgment on auditory inputs. In our experiments, we thus focused on automatic or task-irrelevant novelty detection in the auditory domain (and its plasticity related to musical training), because automaticity is an important property of an efficient novelty discrimination system allowing the brain to rapidly and effortlessly detect change in the environment (Sokolov, 1963; Brown and Bashir, 2002; Yamaguchi et al., 2004; Kumaran and Maguire, 2007b). Note that this approach is similar to experimental conditions commonly used in electrophysiological studies to test for the neural correlates of preattentive oddball detection in the acoustic domain (for review, see Näätänen and Escera, 2000; Näätänen et al., 2001) and to identify functional differences in automatic auditory processing in musicians and nonmusicians on a cortical (for review, see Munte et al., 2002) and subcortical level (Parbery-Clark et al., 2009).

\section{Behavioral testing of musical skills}

To behaviorally assess basic temporal sound processing facilities, subjects of the cross-sectional study performed a standardized test measuring musical abilities after functional imaging, which required the detection of small deviances in short melodies in a forced-choice task [Advanced Measures of Music Audiation (AMMA) test] (Gordon, 1998) (see supplemental Table 1, available at www.jneurosci.org as supplemental material).

For evaluation of aural skills of students in the longitudinal study, they participated in a music dictation, probably the most common measure to evaluate musical aural skills at the university level. At two times (at the beginning at the end of our study period), a piece of music (cut to "takes" of a few seconds) was presented to the students by headphones. The students had to write the musical scores of the soprano and the bass voice. The scores were judged by university teachers at Hochschule fuer Musik by giving credits corresponding to each correct measure in each voice. The achieved and the possible credits of the dictation were converted to a percentage measure of correct answers. The following parts of music have been used for the dictation: (1) W. A. Mozart: symphony KV 319, 2d movement, measures 1-18; (2) J. S. Bach: cantata BWV 119, no. 5 (alto aria), measures 1-13. The degree of difficulty of these two music parts was comparable.

The music dictations took place outside the MR room in the rooms of Hochschule fuer Musik. The students were familiar with the situation of the music dictation. Sixteen (of 19) students participated at the baseline dictation ("Mozart"), and 17 after their two semesters of training (follow-up, "Bach").

\section{Data acquisition}

For the cross-sectional experiment (experiment 1), fMRI data were acquired on a $1.5 \mathrm{~T}$ standard clinical MRI scanner (Siemens) equipped with an Espree gradient system and a circularly polarized radio frequency headcoil. Data from the longitudinal experiment (experiment 2) were acquired with a 3 T MRI scanner (Siemens) equipped with an Allegra gradient system and a circularly polarized frequency headcoil. In all imaging experiments, the subject's head was fixated with foam pads to minimize movement during the experiment. A T1-weighted highresolution data set covering the whole brain was collected for each subject with a three-dimensional magnetization-prepared rapid acquisition gradient echo with $1.2 \times 1 \times 1 \mathrm{~mm}^{3}$ (experiment 1 ), respectively, a threedimensional modified driven equilibrium Fourier transform sequence with $1 \times 1 \times 1 \mathrm{~mm}^{3}$ (experiment 2).

To reduce perceptual and physiological interactions of the blood oxygen level-dependent (BOLD) signal caused by the acoustic noise produced by switching magnetic field gradients in fMRI with activity 
induced by experimental acoustic stimulation, we used a recently developed novel low-impact noise acquisition fMRI sequence, which increases in the dynamic range of BOLD signal (Seifritz et al., 2006) for functional imaging. In short, this sequence elicits a scanner gradient acoustic noise, which is perceived to be continuous. By way of contrast, conventional echoplanar imaging sequences produce a pulsed scanner noise pattern, which could heavily interfere with the temporal structure of the acoustic stimulation used in our study [for detailed illustration of sound envelopes of scanner noise, see Seifritz et al. (2006), their Fig. 1].

The functional volumes were positioned parallel to the lateral sulcus.

Imaging parameters of the cross-sectional experiment (1.5 T). Imaging parameters of the cross-sectional experiment $(1.5 \mathrm{~T})$ were as follows: gradient-recalled echoplanar low-impact noise acquisition imaging sequence with 16 image slices having a thickness of $5 \mathrm{~mm}$ and a volume repetition time (TR) of $1850 \mathrm{~ms}$ (field of view, $180^{2} \mathrm{~mm}^{2}$; matrix, $64^{2}$ pixels; echo time, $61 \mathrm{~ms}$; flip angle, $90^{\circ}$; bandwidth, $1280 \mathrm{~Hz} /$ pixel; slice acquisition time, $116 \mathrm{~ms}$ ).

Imaging parameters of the longitudinal experiment ( $3 \mathrm{~T})$. Imaging parameters of the longitudinal experiment $(3 \mathrm{~T})$ were as follows: gradientrecalled echoplanar low-impact noise acquisition imaging sequence with 20 image slices having a thickness of $4 \mathrm{~mm}$ and a volume TR of $1880 \mathrm{~ms}$ (field of view, $220^{2} \mathrm{~mm}^{2}$; matrix, $64^{2}$ pixels; echo time, $30 \mathrm{~ms}$; flip angle, $90^{\circ}$; bandwidth, $1280 \mathrm{~Hz} /$ pixel; slice acquisition time, $62 \mathrm{~ms}$ ).

\section{Data preprocessing}

Image time courses were processed using the software package BrainVoyager QX (Brain Innovation): for each subject, the first two echoplanar images were discarded to allow for magnetization signal full saturation, and all the remaining scans were realigned to the first included volume scan using a Levenberg-Marquardt algorithm optimizing three translation and three rotation parameters on a resampled version of each image. The resulting head motion-corrected time series were corrected for the different slice scan times using a cubic spline interpolation procedure and then filtered in the temporal domain. For temporal filtering, a highpass filter with cutoff to six cycles per time course (114 s) was used to reduce linear and nonlinear trends in the time courses. Using the results of the image registration with three-dimensional anatomical scans, the functional image-time series were warped into Talairach space and resampled into $3 \mathrm{~mm}$ isotropic voxel time series. Finally, to perform a grouplevel analysis, the resampled volume time series were spatially filtered (smoothing) using a $6 \mathrm{~mm}$ full-width at half-maximum Gaussian kernel.

\section{Statistical analysis}

The variance of all image time series was estimated voxelwise according to a random effects convolution-based general linear model (GLM) analysis (Friston et al., 1995, 1999). Three "event-type" predictors of interest encoding the responses to the three deviant types and one "block-type" predictor of no interest encoding the response to the standard stimulus against a baseline of no auditory stimulation were defined using the double-gamma function (Friston et al., 1998) as hemodynamic input function for the linear convolution. In total, the design matrix included five predictors, three predictors of interest (for the deviant events) and two confounds (for the standard response and the "constant" baseline).

For each subject and each voxel included in the slab of imaging, the five " $\beta$ " weights of the five regressors were estimated according to a GLM fit-refit procedure, which ensured a correction of residual serial correlation in the error terms according to a first-order autoregressive model (Bullmore et al., 1996).

To draw population-level inferences from statistical maps, the three $\beta$ estimates for the predictors of interest at each voxel entered a secondlevel ANOVA with subjects treated as random observations (randomeffects ANOVA). Two different factorial designs were defined for the random-effects ANOVA of the cross-sectional and the longitudinal study data. For the cross-sectional experiment, a two-way ANOVA table was prepared, with one within-subject factor for the "temporal novelty" effect (including three levels for the three deviant types) and one betweensubject factor for the "musicianship" effect (including two levels for the two groups of professional musicians and nonmusicians). For the longitudinal study, a three-way ANOVA table was prepared, with two within- subject factors for the temporal novelty effect (including three levels for the three deviant types) and for the "musical training" effect (including two levels for pretraining and posttraining measurements) and one between-subject factor for the musicianship effect (including two levels for the two groups of musical and nonmusical students). The resulting $F$ maps for the main effects of the task in all groups and both studies, for the two-way interaction "temporal novelty by musicianship" in the crosssectional study and "temporal novelty by training" in the longitudinal study and for the three-way interaction "temporal novelty by musical training by musicianship" in the longitudinal study were overlaid on a Montreal Neurological Institute template brain. To localize the significant effects on the average anatomy, a threshold was applied to the $F$ maps, which protected against false-positive clusters at $5 \%$ (corrected for multiple comparisons). Starting from the uncorrected threshold of $p=$ 0.001, a whole-slab cluster-level correction approach based on Monte Carlo simulations (Forman et al., 1995; Etkin et al., 2004) was used to define the corresponding minimum cluster size to apply. Only clusters that survived this thresholding procedure are reported in Results.

To test for correlations between BOLD responses to temporal novelty and behaviorally assessed musical abilities, a region of interest (ROI) was functionally defined from those voxels that exhibited a statistically significant three-way interaction (temporal novelty by training by musicianship) after the voxel-based analysis of the data from the longitudinal study (experiment 2). To preclude any circularity of data analysis (Kriegeskorte et al., 2009), we then applied this ROI mask derived from experiment 2 (longitudinal study) to the independent fMRI data acquired in experiment 1 (cross-sectional study). The average time courses from these voxels were extracted for each subject (experiment 1) and resubmitted to the same GLM (ROI-GLM). The resulting ROI-GLM fits were finally correlated with individual musical abilities of the subjects as evaluated with the AMMA test (see supplemental Table 1, available at www.jneurosci.org as supplemental material) for the cross-sectional experiment using an analysis of covariance (ANCOVA) model with one categorical factor (musicianship) and one continuous factor (AMMA score).

\section{Results}

\section{Cross-sectional study (experiment 1)}

We presented a temporal mismatch paradigm consisting of regularly spaced sine tones interspersed with infrequently occurring deviant SOAs (Fig. 1) to groups of professional musicians $(n=7)$ and nonmusicians $(n=7)$, matched for age and gender (for detailed subject characterization, see supplemental Table 1, available at www.jneurosci.org as supplemental material), to test for differential brain responses to temporal novelty in the acoustic domain between groups.

The occurrence of standard sine tones, presented with shorter SOAs $(142,130,100 \mathrm{~ms})$ compared with the standard SOA (150 ms) (Fig. 1), elicited a mismatch response in the temporal plane of the right hemisphere $(p<0.05$, corrected $)$, which depended on the degree of deviance. The greater the deviance (i.e., the shorter the deviant SOAs), the more neural activity was induced (Fig. 2A,B). BOLD activity in the right planum temporale induced by a temporal mismatch did not differ significantly between musicians and nonmusicians.

However, a second-level two-way interaction analysis with the factors temporal novelty (three levels of temporal deviance) and musicianship (professional musicians vs nonmusician) revealed enhanced BOLD responses to novelty of temporal structure in musicians' left anterior hippocampus ( $p<0.05$, corrected) (Fig. $2 C$ ) (for details, see supplemental Fig. S2, available at www. jneurosci.org as supplemental material).

\section{Longitudinal study (experiment 2)}

We presented the temporal mismatch paradigm from experiment 1 to an independent sample of music students and stu- 
dents from other faculties $(n=40)$ (supplemental Table 2, available at www. jneurosci.org as supplemental material). To evaluate the impact of academic musical training on temporal novelty detection, BOLD responses to temporal novelty were measured twice in both groups. $\mathrm{Mu}-$ sic students were examined before they started musical training at university level, and after completing the first two semesters at university, which include aural skill training (see supplemental material, available at www.jneurosci.org). In addition, aural skills were quantified behaviorally using a music dictation, to test for changes across time. We used percentage measures to compare the results of the two music dictations [baseline (1) and follow-up (2)] (see Materials and Methods) of music students. Results showed a high interindividual variance. The students achieved a mean of $65.6 \%(20.5-99.6$; SD, 27.2) correct answers in music dictation 1 (Mozart: symphony KV 319, 2d movement, measures 1-18) and 77.3\% (20.7-100; SD, 27.4) in music dictation 2 [J. S. Bach: cantata BWV 119, no. 5 (alto aria), measures 1-13]. Using a Wilcoxon test for nonparametric data revealed a significant time effect (i.e., the music students performed better after one-half year of ear training $)(Z=-2.275$; $p=0.023$, two-tailed).

Students from other faculties, however, did not receive any aural skill training between the two longitudinal evaluation time points in addition to the formal education specific to their individual fields of study.

\section{Deviance detection in the planum temporale of the right hemisphere}

Computing the main effect of deviant SOAs (142, 130, $100 \mathrm{~ms})$ in the cohort of all students participating in the longitudinal study revealed BOLD activity related to novelty in temporal structure in the planum temporale of the right hemisphere, consistent with the results of the cross-sectional study (Fig. 3A). Like in the crosssectional study, the amplitude of the BOLD response in this region depended on the degree of deviance (i.e., the greater the irregularity compared with the standard temporal pattern, the more neural activity is elicited in this region) (Fig. $3 B$ ).

\section{Group differences in temporal novelty detection not related to academic musical training}

Evaluating the impact of musicianship (musical students vs students of other faculties) on temporal novelty detection in the longitudinal sample without considering the factor training (i.e., the impact of academic musical training between the two longitudinal scan sessions separated by academic aural skills training) in a two-way interaction analysis (temporal novelty by group) revealed a significant interaction $(p<0.05$, corrected $)$ in the insula and precuneus of the right hemisphere in response to temporal novelty (supplemental Fig. S1, available at www.jneurosci. org as supplemental material). In these right hemispheric regions, musical students showed enhanced BOLD responses to temporal deviance compared with other students. However, in contrast to the cross-sectional study, this two-way interaction revealed no significant differences in temporal novelty processing in left anterior hippocampus between the groups of the longitudinal sample.

\section{Effect of academic musical training on hippocampal novelty detection}

To test for the effect of academic musical training on brain responses to temporal novelty, we performed a three-way interaction analysis with the factors temporal novelty (three levels of temporal deviance) by musicianship (musical students vs students of other faculties) by training (measurements before and after musical students received general ear training). We found a significant interaction $(p<0.05$, corrected) of the three factors in the left anterior hippocampus: in comparison with students of nonmusical faculties, musical students showed enhanced hippocampal BOLD responses to temporal novelty only after refining their musical skills through training at academic level (Fig. 3C) (for details, see also supplemental Fig. S3, available at www. jneurosci.org as supplemental material). Note that the evaluation of the two-way interaction between the factors temporal novelty and training (without considering the factor musicianship) revealed significant effects in the left inferior frontal gyrus only ( $p<0.05$, corrected), but not in hippocampus. That is, neither the reexposition to the temporal novelty paradigm across groups (factor training) nor the factor musicianship (see above for results of the interaction temporal novelty by musicianship) alone, but only their interaction accounts for the enhanced hippocampal activation, suggesting training-related changes of acoustic novelty detection capabilities in left anterior hippocampus in musical students receiving (task-unrelated) training of musical skills at university.

In summary, like in professional musicians with an academic musical background (see results of cross-sectional study), a general training of aural skills at academic level enhanced BOLD activity in left anterior hippocampus in response to temporal novelty in musical students.

\section{Behavioral correlates of enhanced hippocampal novelty detection in musicians}

We tested for correlations between BOLD responses to temporal novelty in regions showing training-related plasticity (i.e., left hippocampus) and musical aptitude as evaluated using a common standardized measure (AMMA test) (Gordon, 1998). To avoid any circularity of statistical analysis (Kriegeskorte et al., 2009), we used our two independent datasets from experiments 1 and 2 for ROI selection and subsequent analysis. More specifically, first the ROI was functionally defined by the voxel-based three-way interaction analysis with the factors temporal novelty, musicianship, and training in the longitudinal study (experiment $2)$, representing the brain area in which we observed training- 

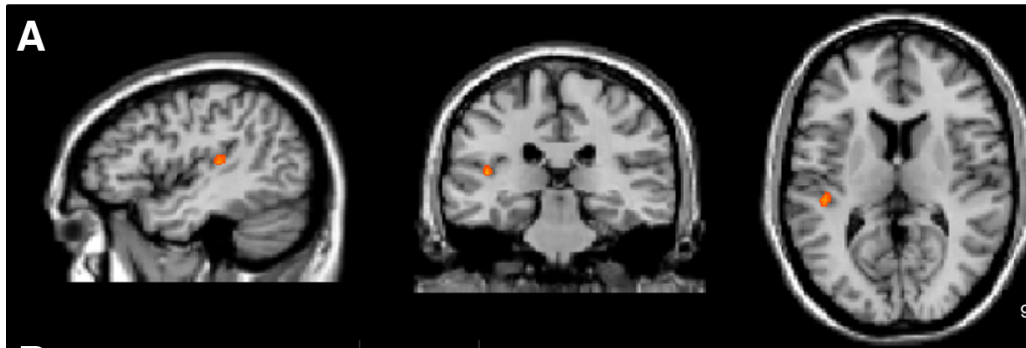

B
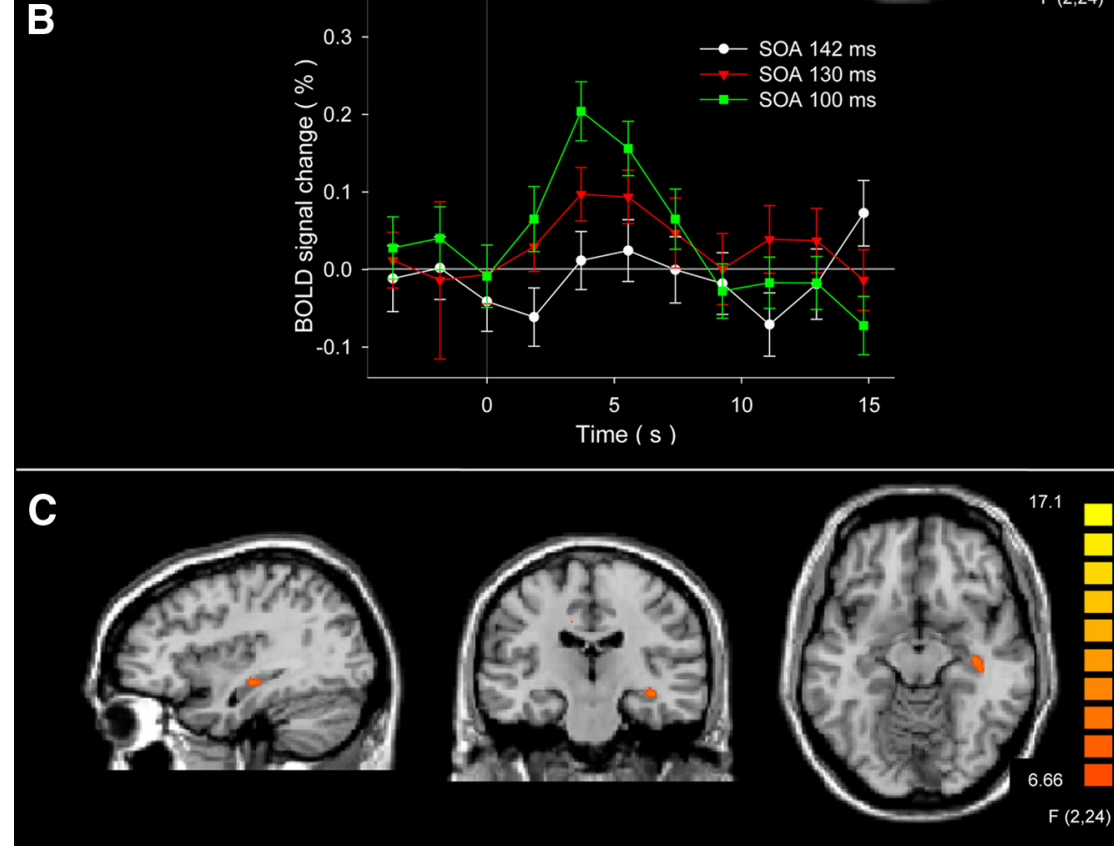

Figure 2. BOLD responses to temporal novelty in the cross-sectional population (experiment 1). $A$, Computing the main effect for all grades of irregular SOAs reveals that deviant events embedded in an otherwise regular temporal pattern induce BOLD activity in planum temporale of the right hemisphere [Talairach coordinates (in $\mathrm{mm}$ ): $x=+52, y=-27, z=+8$ ] in musicians and nonmusicians ( $p<0.05$, cluster level correction). $\boldsymbol{B}$, Event-related averaging of BOLD signal changes in right temporal plane (rPT) elicited by deviant events (SOA of 142, 130, or $100 \mathrm{~ms}$ ) shows a parametric variation of the novelty response according to the different degrees of deviance (i.e., the greater the deviance to the regular pattern the more neural activity in rPT is induced). Error bars indicate SEM. C, Second-level two-way interaction analysis with the factors temporal novelty (three levels of temporal deviance) and musicianship (professional musicians vs nonmusicians) revealed enhanced BOLD responses to novelty of temporal structure in musicians' left anterior hippocampus $(x=-35, y=-19, z=-8 ; p<0.05$, corrected).

related changes (left hippocampus) (Fig. 3). Then, using independent data from subjects of experiment 1 , we found that BOLD responses to temporal novelty within this ROI are correlated with the individuals' musical abilities as evaluated separately using the AMMA test (ANCOVA, $F=4.81, p=0.05$ ), pointing to the behavioral significance of the functional differences found in musicians' hippocampus.

\section{Discussion}

Deviance detection in the planum temporale of the right hemisphere

We found that right-hemispheric secondary auditory cortex is crucially involved in detection of temporal pattern variations embedded in an otherwise temporally regular sequence of tones. The planum temporale has been previously suggested to be a "computational hub," which is essential for segregating spectrotemporal sound patterns and matching them with stored representations (Griffiths and Warren, 2002), and mismatches in the temporal domain seem to be preferentially processed in the right hemisphere (Mustovic et al., 2003; Herdener et al., 2007). The modulation of BOLD response amplitude with degree of deviance in

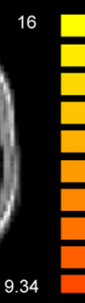

$F(2,24)$

this region is consistent with electrophysiological data showing a parametric variation of oddball induced activity with deviance modulation (Näätänen et al., 2001). However, BOLD activity to temporal mismatch in this region does not differ between groups of musicians and nonmusicians, neither in our cross-sectional comparison of professional musicians versus nonmusicians (experiment 1) nor in our longitudinal study evaluating the impact of musical training on acoustic novelty detection (experiment 2). This indicates a comparable detection of temporal novelty in secondary auditory regions of both musicians and nonmusicians.

\section{Enhanced novelty detection in left anterior hippocampus in musical experts}

Significant group differences in the crosssectional comparison (experiment 1) of temporal novelty detection in the auditory domain were observed in the anterior left hippocampus, which shows enhanced BOLD response to temporal irregularities in professional musicians compared with nonmusicians. Therefore, our data suggest that, although secondary auditory cortex is crucially involved in the preattentive processing of temporal acoustic patterns, differential activation of the left anterior hippocampus represents a functional correlate of musical expertise related to sound pattern processing. This notion is corroborated by the correlation of musical aptitude measures with hippocampal BOLD responses.

The hippocampus has been previously suggested to be involved in the encoding of time intervals (Knight et al., 2004), and in particular anterior regions of the left hippocampus formation in humans have been proposed to index novelty (Strange et al., 1999). Furthermore, the ability to act as a relational operator enabling comparisons between data input and stored data and thereby detecting changes and novel events in the sensory environment has been previously attributed to the hippocampus based on theoretical (Gray and Rawlins, 1986) and animal (Vinogradova, 2001) models and is pivotal when dealing with the recognition of deviant temporal variations embedded in an otherwise regular stimulus pattern. It is also possible that, in analogy to the processing of novelty in the visual domain, the hippocampus is capable of extracting the probabilistic temporal structure of acoustic streams and thus represents the expected information or novelty of any event within a stimulation stream before it occurs (cf. Strange et al., 2005). A recent fMRI study in humans found left hippocampal activation in response to associative novelty, which was investigated by varying the temporal order within a sequence of visual stimuli (Kumaran and Maguire, 2006). Here, we observed that time interval variation within an otherwise regular temporal pattern can induce hippocampal activity. Our data thus support the idea that the hippocampus acts as a novelty detector by compar- 
ing actual sensory inputs (e.g., irregularly spaced sine tones) with immediately previous inputs (e.g., preceding periods of acoustic inputs with a regular temporal pattern building up a regular representation or expectation of the acoustic environment), generating novelty signals when previous predictions are violated by sensory reality (Kumaran and Maguire, 2007a), and extend it further to the temporal domain by showing that the hippocampus is also sensitive to variations of time intervals.

\section{Enhanced hippocampal novelty detection in musicians: talent or training?}

The question remains, however, whether exposure (Monaghan et al., 1998) to music, a genetic predisposition (Thompson et al., 2001), or both contribute to the functional differences observed in our cross-sectional study. We thus tested for the impact of musical training at an academic level on hippocampal properties related to temporal novelty detection in a longitudinal approach, and investigated students of the local academy of music before and after receiving ear training during the first semesters of their studies compared with students of other faculties (experiment 2).

First, we specified functional differences in brain responses to temporal novelty between groups of the longitudinal sample without considering trainingrelated effects. We found a significant two-way interaction of the factors temporal novelty by musicianship in the right insular region and the precuneus, pointing to functional differences in these regions between musical and nonmusical students. The right anterior insula has been implicated in the detection of visual and auditory temporal pattern changes (Herdener et al., 2009) and in the processing of novelty tested with various visual, tactile, and auditory oddball paradigms (Linden et al., 1999; Ardekani et al., 2002; Downar et al., 2002). The implication of the right precuneus in novelty detection has also been reported recently (Gur et al., 2007). Functional differences that we found between groups in these regions might be associated with musical talent and/or preacademic musical training. It is important to note, however, that this two-way interaction analysis with the factors temporal novelty by musicianship (and without considering the effect of academic musical training between the two longitudinal sessions) did not reveal any significant functional differences between groups of students $(n=40)$ in the hippocampus. This is in contrast to the results of the cross-sectional study, in which professional musicians showed enhanced hippocampal responses to temporal novelty when compared with nonmusicians $(n=14)$. This suggests that neither the extensive preacademic musical experience of musical students before their education on an academic level nor talent/genetic pre- disposition is sufficient to account for differential hippocampal novelty detection.

The activation of the left inferior frontal gyrus in response to temporal novelty as revealed by the two-way interaction analysis with the factors temporal novelty by training is consistent with previous electrophysiological studies showing an involvement of inferior frontal regions in the detection of changes of various acoustic stimulus features (Deouell et al., 1998; Rinne et al., 2000; Opitz et al., 2002). More specifically, this region has been suggested to be especially sensitive to variations of sounds in the temporal domain (Molholm et al., 2005), and our data thus support the notion of inferior frontal gyrus as a detector of temporal acoustic oddballs. However, activity in this region did not differ between groups.

In a next step, we specified the effects of academic musical training on brain responses to temporal novelty in a whole-brain interaction analysis of the longitudinal data with the three factors temporal novelty by musicianship by training. Considering training-related plasticity in students, we found a significant, whole-brain-corrected three-way interaction $(p<0.05$, corrected) in left anterior hippocampus (but not in any other brain 
region) (Fig. 3C) with enhanced BOLD activity in musical students after they had received academic ear training. This finding demonstrates that musical students show enhanced BOLD activity to temporal novelty in the same region (i.e., left anterior hippocampus) where we found functional differences between professional musicians, who were all experts in ear training, and nonmusicians only after receiving academic ear training, suggesting that we were able to identify functional correlates of traininginduced changes in sound pattern processing.

In addition, we found a correlation of hippocampal sensitivity to temporal novelty with musical abilities. Based on our imaging and behavioral data, we assume that the observed changes in hippocampal activity in musicians represent a functional correlate of a tuning of aural skills related to time interval perception during the course of their studies. There is also evidence showing that lesions of the left hippocampus in humans impair performance related to time interval discrimination within otherwise regular acoustic streams similar to the sounds presented in our study (Samson et al., 2001), supporting the behavioral relevance of the left hippocampus for temporal novelty detection in the acoustic modality.

Taken together, our data extend the notion of the hippocampus as a novelty detector to the temporal domain of the acoustic modality and expand previous findings on the impact of musical expertise on brain functions (Elbert et al., 1995; Pantev et al., 1998; Schneider et al., 2002; Parbery-Clark et al., 2009) to the hippocampal region. Moreover, to our knowledge, this is the first longitudinal investigation showing that training can induce functional plasticity in the adult human hippocampus, as exemplified using musicians' brains as a model.

\section{References}

Ardekani BA, Choi SJ, Hossein-Zadeh GA, Porjesz B, Tanabe JL, Lim KO, Bilder R, Helpern JA, Begleiter H (2002) Functional magnetic resonance imaging of brain activity in the visual oddball task. Brain Res Cogn Brain Res 14:347-356.

Brown MW, Bashir ZI (2002) Evidence concerning how neurons of the perirhinal cortex may effect familiarity discrimination. Philos Trans R Soc Lond B Biol Sci 357:1083-1095.

Bullmore E, Brammer M, Williams SC, Rabe-Hesketh S, Janot N, David A, Mellers J, Howard R, Sham P (1996) Statistical methods of estimation and inference for functional MR image analysis. Magn Reson Med 35:261-277.

Deouell LY, Bentin S, Giard MH (1998) Mismatch negativity in dichotic listening: evidence for interhemispheric differences and multiple generators. Psychophysiology 35:355-365.

Downar J, Crawley AP, Mikulis DJ, Davis KD (2002) A cortical network sensitive to stimulus salience in a neutral behavioral context across multiple sensory modalities. J Neurophysiol 87:615-620.

Draganski B, Gaser C, Kempermann G, Kuhn HG, Winkler J, Büchel C, May A (2006) Temporal and spatial dynamics of brain structure changes during extensive learning. J Neurosci 26:6314-6317.

Ekstrom AD, Kahana MJ, Caplan JB, Fields TA, Isham EA, Newman EL, Fried I (2003) Cellular networks underlying human spatial navigation. Nature 425:184-188.

Elbert T, Pantev C, Wienbruch C, Rockstroh B, Taub E (1995) Increased cortical representation of the fingers of the left hand in string players. Science 270:305-307.

Etkin A, Klemenhagen KC, Dudman JT, Rogan MT, Hen R, Kandel ER, Hirsch J (2004) Individual differences in trait anxiety predict the response of the basolateral amygdala to unconsciously processed fearful faces. Neuron 44:1043-1055.

Forman SD, Cohen JD, Fitzgerald M, Eddy WF, Mintun MA, Noll DC (1995) Improved assessment of significant activation in functional magnetic resonance imaging (fMRI): use of a cluster-size threshold. Magn Reson Med 33:636-647.
Friston KJ, Holmes AP, Poline JB, Grasby PJ, Williams SC, Frackowiak RS, Turner R (1995) Analysis of fMRI time-series revisited. Neuroimage 2:45-53.

Friston KJ, Josephs O, Rees G, Turner R (1998) Nonlinear event-related responses in fMRI. Magn Reson Med 39:41-52.

Friston KJ, Holmes AP, Worsley KJ (1999) How many subjects constitute a study? Neuroimage 10:1-5.

Gordon EE (1998) Introduction to research and psychology of music. Chicago: GIA.

Gray JA, Rawlins JNP (1986) Comparator and buffer memory: an attempt to integrate two models of hippocampal function. In: The hippocampus, pp 159-201. New York: Plenum.

Griffiths TD, Warren JD (2002) The planum temporale as a computational hub. Trends Neurosci 25:348-353.

Gur RC, Turetsky BI, Loughead J, Waxman J, Snyder W, Ragland JD, Elliott MA, Bilker WB, Arnold SE, Gur RE (2007) Hemodynamic responses in neural circuitries for detection of visual target and novelty: an eventrelated fMRI study. Hum Brain Mapp 28:263-274.

Herdener M, Esposito F, Di Salle F, Lehmann C, Bach DR, Scheffler K, Seifritz E (2007) BOLD correlates of edge detection in human auditory cortex. Neuroimage 36:194-201.

Herdener M, Lehmann C, Esposito F, di Salle F, Federspiel A, Bach DR, Scheffler K, Seifritz E (2009) Brain responses to auditory and visual stimulus offset: shared representations of temporal edges. Hum Brain Mapp 30:725-733.

Kempermann G, Kuhn HG, Gage FH (1997) More hippocampal neurons in adult mice living in an enriched environment. Nature 386:493-495.

Knight DC, Cheng DT, Smith CN, Stein EA, Helmstetter FJ (2004) Neural substrates mediating human delay and trace fear conditioning. J Neurosci 24:218-228.

Knight R (1996) Contribution of human hippocampal region to novelty detection. Nature 383:256-259.

Kriegeskorte N, Simmons WK, Bellgowan PS, Baker CI (2009) Circular analysis in systems neuroscience: the dangers of double dipping. Nat Neurosci 12:535-540.

Kumaran D, Maguire EA (2006) An unexpected sequence of events: mismatch detection in the human hippocampus. PLoS Biol 4:e424.

Kumaran D, Maguire EA (2007a) Which computational mechanisms operate in the hippocampus during novelty detection? Hippocampus 17 : 735-748.

Kumaran D, Maguire EA (2007b) Match mismatch processes underlie human hippocampal responses to associative novelty. J Neurosci 27:8517-8524.

Linden DE, Prvulovic D, Formisano E, Völlinger M, Zanella FE, Goebel R, Dierks T (1999) The functional neuroanatomy of target detection: an fMRI study of visual and auditory oddball tasks. Cereb Cortex 9:815-823.

Lledo PM, Alonso M, Grubb MS (2006) Adult neurogenesis and functional plasticity in neuronal circuits. Nat Rev Neurosci 7:179-193.

Maguire EA (2001) Neuroimaging, memory and the human hippocampus. Rev Neurol (Paris) 157:791-794.

Maguire EA, Gadian DG, Johnsrude IS, Good CD, Ashburner J, Frackowiak RS, Frith CD (2000) Navigation-related structural change in the hippocampi of taxi drivers. Proc Natl Acad Sci U S A 97:4398-4403.

Molholm S, Martinez A, Ritter W, Javitt DC, Foxe JJ (2005) The neural circuitry of pre-attentive auditory change-detection: an fMRI study of pitch and duration mismatch negativity generators. Cereb Cortex 15:545551.

Monaghan P, Metcalfe NB, Ruxton GD (1998) Does practice shape the brain? Nature 394:434.

Munte TF, Altenmuller E, Jancke L (2002) The musician's brain as a model of neuroplasticity. Nat Neurosci 3:473-478.

Mustovic H, Scheffler K, Di Salle F, Esposito F, Neuhoff JG, Hennig J, Seifritz E (2003) Temporal integration of sequential auditory events: silent period in sound pattern activates human planum temporale. Neuroimage 20:429-434.

Näätänen R, Escera C (2000) Mismatch negativity: clinical and other applications. Audiol Neurootol 5:105-110.

Näätänen R, Tervaniemi M, Sussman E, Paavilainen P, Winkler I (2001) "Primitive intelligence" in the auditory cortex. Trends Neurosci 24:283-288.

Opitz B, Rinne T, Mecklinger A, von Cramon DY, Schröger E (2002) Dif- 
ferential contribution of frontal and temporal cortices to auditory change detection: fMRI and ERP results. Neuroimage 15:167-174.

Pantev C, Oostenveld R, Engelien A, Ross B, Roberts LE, Hoke M (1998) Increased auditory cortical representation in musicians. Nature 392: 811-814.

Parbery-Clark A, Skoe E, Kraus N (2009) Musical experience limits the degradative effects of background noise on the neural processing of sound. J Neurosci 29:14100-14107.

Peretz I (2006) The nature of music from a biological perspective. Cognition $100: 1-32$.

Rinne T, Alho K, Ilmoniemi RJ, Virtanen J, Näätänen R (2000) Separate time behaviors of the temporal and frontal mismatch negativity sources. Neuroimage 12:14-19.

Rüsseler J, Altenmüller E, Nager W, Kohlmetz C, Münte TF (2001) Eventrelated brain potentials to sound omissions differ in musicians and nonmusicians. Neurosci Lett 308:33-36.

Samson S, Ehrle N, Baulac M (2001) Cerebral substrates for musical temporal processes. Ann N Y Acad Sci 930:166-178.

Schneider P, Scherg M, Dosch HG, Specht HJ, Gutschalk A, Rupp A (2002) Morphology of Heschl's gyrus reflects enhanced activation in the auditory cortex of musicians. Nat Neurosci 5:688-694.

Seifritz E, Di Salle F, Esposito F, Herdener M, Neuhoff JG, Scheffler K (2006)
Enhancing BOLD response in the auditory system by neurophysiologically tuned fMRI sequence. Neuroimage 29:1013-1022.

Sokolov EN (1963) Higher nervous functions; the orienting reflex. Annu Rev Physiol 25:545-580.

Strange BA, Dolan RJ (2001) Adaptive anterior hippocampal responses to oddball stimuli. Hippocampus 11:690-698.

Strange BA, Fletcher PC, Henson RN, Friston KJ, Dolan RJ (1999) Segregating the functions of human hippocampus. Proc Natl Acad Sci U S A 96:4034-4039.

Strange BA, Duggins A, Penny W, Dolan RJ, Friston KJ (2005) Information theory, novelty and hippocampal responses: unpredicted or unpredictable? Neural Netw 18:225-230.

Thompson PM, Cannon TD, Narr KL, van Erp T, Poutanen VP, Huttunen M, Lönnqvist J, Standertskjöld-Nordenstam CG, Kaprio J, Khaledy M, Dail R, Zoumalan CI, Toga AW (2001) Genetic influences on brain structure. Nat Neurosci 4:1253-1258.

Vinogradova OS (2001) Hippocampus as comparator: role of the two input and two output systems of the hippocampus in selection and registration of information. Hippocampus 11:578-598.

Yamaguchi S, Hale LA, D’Esposito M, Knight RT (2004) Rapid prefrontal-hippocampal habituation to novel events. J Neurosci 24: 5356-5363. 